\title{
Efeito dos antidepressivos ISRS sobre os hormônios tireoidianos
}

\author{
SSRI antidepressant effects on thyroid hormones
}

Saint-Clair Bahls', Gisah Amaral de Carvalho', Anke Boeving'

\section{RESUMO}

O objetivo deste artigo é realizar uma atualização sobre a ação de antidepressivos, com destaque aos inibidores seletivos de recaptação de serotonina (ISRS) na função tireoidiana de pacientes com depressão. Sete ensaios clínicos investigaram o efeito dos ISRS sobre a função tireoidiana. Apesar das diferenças metodológicas, o principal achado foi a tendência à diminuição dos níveis plasmáticos de tiroxina, não necessariamente relacionada com a resposta clínica, e sem efeito sobre a tireotropina na maioria das pesquisas. Os estudos sugerem que os ISRS promovem efeitos na função tireoidiana em alguns pacientes com depressão, especificamente diminuição nos níveis plasmáticos de tiroxina. Porém, observou-se que a relação entre o uso de antidepressivos ISRS e a função tireoidiana não está

\section{Palavras-chave}

Função tireoidiana, ISRS, transtornos depressivos.

\section{Key-words}

Thyroid function, SSRIs, depressive disorders. suficientemente esclarecida. Mesmo nos casos de alteração nos níveis plasmáticos dos hormônios tireoidianos em resposta a ação dos ISRS, esta pode ser uma ação não específica sobre a função tireoidiana.

\begin{abstract}
This article aims at updating antidepressant action, especially using selective serotonin reuptake inhibitors, on thyroid function in depressed patients. Seven clinical trials investigated the status of thyroid hormones after treatment with SSRIs. Despite methodological differences, the main finding indicated a tendency towards decreased serum thyroxine levels, The majority of studies could not find a positive relationship between lower serum thyroxine level and a favorable treatment response. Also, an effect on thyrotropin could not be found. Those study results suggest SSRIs promote effects on thyroid function in some depressed patients, specifically decreased serum thyroxine levels. However, the relation between SSRIs antidepressant use and thyroid function is not clear. Even when there was a change in serum thyroid hormone levels due to SSRI therapy, this could be a non-specific effect on thyroid function.
\end{abstract}

1 Serviço de Endocrinologia e Metabologia do Hospital de Clínicas da Universidade Federal do Paraná (SEMPR). Departamento de Clínica Médica da UFPR 


\section{INTRODUÇÃO}

A relevância da relação entre a função tireoidiana e a depressão foi destacada em recente revisão, na qual observamos que a condição tireoidiana exerce influência importante nas vias cerebrais de sinalização intracelular e que mudanças dos níveis de seus hormônios podem apresentar efeitos diretos no funcionamento cerebral (Bahls e Carvalho, 2004).

As investigações sobre alterações no eixo hipotálamohipófise-tireóide (HHT) em pacientes com depressão produziram achados que podem ser assim resumidos: a) aumento do nível plasmático de tiroxina (T4) em 20\% a 30\% dos casos; b) aumento do nível plasmático de anticorpos antitireoidianos em 15\% dos casos; c) resposta diminuída da tireotropina (TSH) ao teste do hormônio liberador de tireotropina (TRH) em 25\% dos casos; e d) aumento no líquido cefalorraquidiano de TRH (Bahls e Carvalho, 2004). Apesar de alguns autores destacarem que estes achados podem não ser específicos da depressão (Joffe e Sokolov, 1994; Baumgartner, 2000), estas alterações indicam uma interface importante para, pelo menos, uma parcela dos pacientes deprimidos.

Quanto ao emprego dos antidepressivos tricíclicos (ADT) no tratamento da depressão maior (DM) e sua ação sobre a função tireoidiana, o efeito mais comumente encontrado tem sido a redução de T4T e/ou de tiroxina livre (T4L) (Whybrow et al., 1972; Kirkegaard et al., 1977; Bech et al., 1978; Langer et al., 1980; Unden et al., 1986; Baumgartner et al., 1988; Muller e Boning, 1988; Brady; Anton, 1989; Joffe; Singer, 1990; Höflich et al., 1992; Brady et al., 1994; Duval et al., 1996; Rao et al., 1996; Gendall et al., 2003). Esta diminuição de T4 e T4L, apesar de significativa, permanece dentro dos limites da normalidade e ocorre na recuperação do quadro clínico.

$\mathrm{Na}$ atualidade, o emprego dos antidepressivos Inibidores Seletivos de Recaptação de Serotonina (ISRS) é cada vez maior, e pouco se conhece sobre sua ação nos hormônios da tireóide $(H T)$. Esta atualização objetivou examinar os estudos existentes sobre estes antidepressivos, na função tireoidiana de pacientes com depressão. Foi empregado o sistema Medline, no período de 1966 a 2006, com as seguintes palavras-chave: ISRS, depressão maior e hormônios tireoidianos.

\section{O efeito dos ISRS nos HT de pacientes deprimidos}

\section{Fluvoxamina}

Höflich et al. (1992) investigaram o efeito da fluvoxamina e da maprotilina em 41 pacientes com quadros de depressão (38 unipolares e 3 bipolares), durante quatro semanas. Ambos os medicamentos produziram efeitos idênticos sobre os HT. Não houve alterações significativas nos níveis plasmáticos de T3, enquanto o T4T sofreu redução significativa e o TSH apresentou aumento significativo. Também não ocorreram diferenças entre os grupos de respondedores e não-respondedores.

Brady et al. (1994) avaliaram o efeito da fluvoxamina, da imipramina e do placebo em 33 pacientes com DM durante seis semanas. Não houve alterações nos HT (T3, T4 e TSH) com fluvoxamina e com placebo, enquanto com a imipramina ocorreu diminuição significativa de T3 e T4. Foi demonstrada, com a imipramina, uma relação entre a resposta clínica e diminuição significativa nos HT.

\section{Fluoxetina}

Shelton et al. (1993) examinaram 39 pacientes ambulatoriais com DM que receberam fluoxetina e desipramina, em dois momentos, com três e seis semanas de medicamento. Encontraram diminuição significativa de T3, com três semanas, no grupo todo, que não foi mantida na avaliação de seis semanas. Houve associação significativa entre diminuição de T3 e a resposta à fluoxetina, mas não à desipramina. Entre os dois grupos ocorreu aumento pequeno, porém significativo do T4 em seis semanas no grupo desipramina, quando comparado ao grupo fluoxetina Os autores concluíram que não houve alterações significativas nos HT ou no eixo HHT com os antidepressivos.

Gendall et al. (2003) avaliaram a fluoxetina e a nortriptilina em 95 pacientes com DM, durante seis semanas. Encontraram diminuição nos níveis plasmáticos de T4 e T4L, que alcançou diferença significativa somente nos respondedores. Não houve alteração nos níveis de TSH com os medicamentos, e não foram apresentados dados referentes ao T3.

Gitlin et al. (2004) investigaram em 17 pacientes com DM o uso de fluoxetina e de sertralina, durante dez semanas. Identificaram diminuição significativa de T3 e T4 com os medicamentos e não encontraram alteração do T4L e TSH. Não obtiveram diferenças nos níveis plasmáticos dos HT entre respondedores e não-respondedores.

\section{Sertralina}

Dois artigos examinaram a sertralina, porquanto um deles (Gitlin et al., 2004) já foi descrito anteriormente.

Sagud et al. (2002) investigaram 15 pacientes com DM que receberam sertralina e que foram comparados com um grupo controle $(n=16)$, com avaliações em quatro e 24 semanas. O T3 não sofreu alteração com quatro semanas e estava significativamente aumentado no grupo com depressão em 24 semanas, o T4 e o TSH não apresentaram alteração nas duas avaliações. O T4L não foi avaliado. Não houve co-relação significativa entre os escores de depressão e os HT.

\section{Paroxetina}

König et al. (2000) avaliaram em 25 pacientes com DM o efeito da paroxetina em período de quatro semanas. Encontraram diminuição significativa de T4 e não houve 
alteração em T3 e TSH. Houve redução estatisticamente significativa nos níveis de TSH nos não-respondedores. Com o T4 não ocorreu diferença entre respondedores e não-respondedores.

\section{DISCUSSÃO}

As pesquisas envolveram quatro ISRS (fluvoxamina, fluoxetina, sertralina e paroxetina) e obtiveram os seguintes resultados: 1) TSH: seis artigos sem alterações (Shelton et al., 1993; Brady et al., 1994; König et al., 2000; Sagud et al., 2002; Gendall et al., 2003; Gitlin et al., 2004) e um artigo com aumento significativo (Höflich et al., 1992); 2) T3: três artigos sem alterações (Höflich et al., 1992; Brady et al., 1994; König et al., 2000), um com diminuição significativa (Gitlin et al., 2004) e dois com alterações tempo-dependentes, sendo um com diminuição significativa (Shelton et al., 1993) (fluoxetina - diminuição significativa com três semanas que desapareceu em seis semanas) e outro com aumento significativo (Sagud et al., 2002) (sertralina - sem alterações em quatro semanas e com aumento significativo em 24 semanas); 3) T4: quatro artigos sem alterações (Shelton et al., 1993; Brady et al., 1994; Sagud et al., 2002; Gendall et al., 2003), um dos quais apresentou diminuição significativa só nos respondedores (Gendall et al., 2003) e três com diminuição significativa (Höflich et al., 1992; König et al., 2000; Gitlin et al., 2004); 4) T4L: três artigos sem alterações (Shelton et al., 1993; Gendall et al., 2003; Gitlin et al., 2004), um dos quais apresentou diminuição significativa só nos respondedores (Gendall et al., 2003).

Quanto ao aspecto de achados relacionados à resposta clínica, pela análise comparativa entre pacientes respondedores e não-respondedores aos ISRS conclui-se que: quatro artigos não obtiveram diferenças entre os grupos (Höflich et al., 1992; Brady et al., 1994; Sagud et al., 2002; Gitlin et al.,
2004), uma pesquisa identificou diminuição significativa de T3 em resposta à fluoxetina em seis semanas (Shelton et al., 1993), outra pesquisa encontrou diminuição significativa de TSH nos não-respondedores à paroxetina em quatro semanas (König et al., 2000) e uma outra pesquisa identificou diminuição significativa de T4 e T4L nos respondedores à fluoxetina em seis semanas (Gendall et al., 2003). Os resultados com os ISRS encontram-se resumidos na Tabela 1.

Não foram observadas modificações em seis de sete estudos em relação ao TSH, em três de seis estudos com T3, em todos os três estudos com T4L e em quatro de sete estudos com T4. Portanto, considerando os pacientes deprimidos como um todo os resultados indicam que a maioria deles não apresenta alterações nos HT com o uso dos antidepressivos ISRS. Confirmando estas observações, destacamos que a única pesquisa que utilizou um grupo placebo (Brady et al., 1994) não obteve diferença significativa no efeito sobre os HT entre placebo e o ISRS fluvoxamina.

Em razão de algumas pesquisas terem identificado resultados positivos, é provável que um subgrupo de pacientes com depressão apresente alteração nos níveis plasmáticos dos HT na vigência de terapia com ISRS. Nestes casos, a tiroxina parece ser o HT mais suscetível a sofrer modificações, com probabilidade de diminuição de seu nível sérico, o que foi observado em três (Höflich et al., 1992; König et al., 2000; Gitlin et al., 2004) dos sete estudos. Estes estudos também demonstraram diminuição significativa de T3 (Gitlin et al., 2004) e aumento significativo de TSH (Höflich et al., 1992). Estas alterações costumam ocorrer dentro da faixa normal de variação dos hormônios. Todavia, não existe, até o momento, condições de se estabelecer um perfil de quais seriam os deprimidos com mais chances de apresentar alterações nos HT. Apesar de tentadora a hipótese, derivada dos estudos com ADT, de que a diminuição de T4 seria mais comumente encontrada naqueles pacientes respondedo-

Tabela 1. Efeito dos antidepressivos ISRS sobre os HT

\begin{tabular}{|c|c|c|c|c|c|c|c|c|c|}
\hline Autor(es) & $\mathrm{N}^{\circ}$ & Avaliações & Linha de base & $A D$ & T3 & $\mathrm{T} 4$ & T4L & TSH & Resp./ Não-resp. \\
\hline $\begin{array}{l}\text { Höflich et al., } 1992 \\
\text { (Maprotilina) }\end{array}$ & $41 \mathrm{depr}$. & 4 semanas & - & Fluvoxamina & $\operatorname{sem} \neq$ & Dim. sign. & - & Aum. sign. & $\operatorname{sem} \neq$ \\
\hline $\begin{array}{l}\text { Shelton et al., } 1993 \\
\text { (Desipramina) }\end{array}$ & $39 \mathrm{DM}$ & $\begin{array}{l}3 \text { semanas } \\
6 \text { semanas }\end{array}$ & $\begin{array}{c}26 \% \\
\text { Alterações }\end{array}$ & Fluoxetina & $\begin{array}{l}\text { Dim. sign. } \\
\text { sem } \neq\end{array}$ & $\begin{array}{l}\operatorname{sem} \neq \\
\operatorname{sem} \neq\end{array}$ & $\begin{array}{l}\operatorname{sem} \neq \\
\text { sem } \neq\end{array}$ & $\begin{array}{l}\operatorname{sem} \neq \\
\operatorname{sem} \neq\end{array}$ & $\begin{array}{l}\text { Dim. sign. } \\
\text { T3 } \\
\text { Resp./Fluox. }\end{array}$ \\
\hline $\begin{array}{l}\text { Brady et al., } 1994 \\
\text { (Imipramina e Pbo.) }\end{array}$ & $33 \mathrm{DM}$ & 6 semanas & Normal & Fluvoxamina & $\operatorname{sem} \neq$ & sem $\neq$ & - & sem $\neq$ & sem $\neq$ \\
\hline König et al., 2000 & $25 \mathrm{DM}$ & 4 semanas & - & Paroxetina & $\operatorname{sem} \neq$ & Dim. sign. & - & sem $\neq$ & Dim. sign.TSH/Não-resp. \\
\hline \multirow[t]{2}{*}{ Sagud et al., 2002} & $15 \mathrm{DM}+$ & 4 semanas & T3 Dim. sign. & & $\operatorname{sem} \neq$ & $\operatorname{sem} \neq$ & - & $\operatorname{sem} \neq$ & \\
\hline & $\stackrel{\times}{\times}$ & 24 semanas & gr. deprimidas & Sertralina & $\begin{array}{l}\text { Aum. sign. } \\
\text { deprimidas }\end{array}$ & sem $\neq$ & - & $\operatorname{sem} \neq$ & $\operatorname{sem} \neq$ \\
\hline $\begin{array}{l}\text { Gendall et al., } 2003 \\
\text { (Nortriptilina) }\end{array}$ & $95 \mathrm{DM}$ & 6 semanas & sem alterações & Fluoxetina & - & $\begin{array}{l}\text { Dim. sign. } \\
\text { Respond. }\end{array}$ & $\begin{array}{l}\text { Dim. sign. } \\
\text { Respond. }\end{array}$ & sem $\neq$ & $\begin{array}{l}\text { Dim. sign. } \\
\text { T4 e T4L Resp. }\end{array}$ \\
\hline Gitlin et al., 2004 & $17 \mathrm{DM}$ & 10 semanas & - & $\begin{array}{l}\text { Fluoxetina } \\
\text { Sertralina }\end{array}$ & $\begin{array}{l}\text { Dim. sign. } \\
\text { Dim. sign. }\end{array}$ & $\begin{array}{l}\text { Dim. sign. } \\
\text { Dim. sign. }\end{array}$ & $\begin{array}{l}\text { sem } \neq \\
\text { sem } \neq\end{array}$ & $\begin{array}{l}\operatorname{sem} \neq \\
\operatorname{sem} \neq\end{array}$ & sem $\neq$ \\
\hline
\end{tabular}


res aos medicamentos, este achado foi observado somente em uma pesquisa (Gendall et al., 2003) e não confirmado em outras quatro investigações com os ISRS (Höflich et al., 1992; Brady et al., 1994; Sagud et al., 2002; Gitlin et al., 2004).

Sagud et al. (2002) obtiveram um interessante efeito sobre os HT com o uso de sertralina em deprimidos. Avaliando em dois diferentes momentos, encontraram aumento significativo de T3 com 24 semanas de uso, que não havia ocorrido na avaliação com quatro semanas. Esta alteração tempo-dependente levou-os a propor que as alterações com antidepressivos sobre os HT ocorreriam na vigência de tratamento prolongado. Entretanto, alguns dados contradizem este achado, nas duas outras pesquisas que identificaram alterações de T3, uma (Shelton et al., 1993) obteve diminuição significativa com três semanas que regularizou na avaliação de seis semanas, e outra (Gitlin et al., 2004) ocorreu também diminuição significativa com dez semanas. Existe um relato de caso (Harel et al., 1995) em adolescente que após 18 meses de uso de sertralina apresentou baixos níveis de T4, com níveis normais de T3, T4L e TSH, e resposta normal ao teste do TRH. De qualquer forma, fica em aberto esta possibilidade, uma vez que os outros estudos apresentaram menor tempo de acompanhamento, entre quatro a dez semanas de uso de antidepressivos ISRS.

Alguns autores (Gitlin et al., 2004) sugerem que a presença de uma hiperfunção tireoidiana pode predizer a resposta à terapia com ISRS, pois encontraram que o grau de melhora foi relacionado signitivamente com níveis séricos baixos (dentro dos valores normais) de TSH. Gendall et al. (2003) observaram nos pacientes com resposta de achatamento ao teste do TRH uma melhor resposta à fluoxetina do que à nortriptilina, talvez indicando uma alteração predominantemente serotoninérgica nestes pacientes. Existem evidências de que animais hipotireoideos têm síntese de serotonina (5HT) cerebral reduzida (Singhal et al., 1975) e que humanos com hipotireoidismo têm responsividade serotoninérgica reduzida (Bauer et al., 2002), indicando uma relação entre a função tireoidiana e a ação serotoninérgica cerebral. Porém, a exata dimensão desta relação ainda não está esclarecida, uma vez que a atividade do eixo HHT parece estar sob complexa influência do sistema serotoninérgico central (Duval et al., 1999).

Outros autores (Baumgartner et al., 1988; Shelton et al., 1993) propõem que os efeitos nos HT são diferentes entre ISRS e ADT. Baumgartner et al. (1988) encontraram redução de T3 com o ADT serotoninérgico clomipramina e não com o ADT noradrenérgico maprotilina, e sugeriram que os antidepressivos serotoninérgicos podem apresentar um efeito específico de redução do T3.

Como já foi demonstrado com os ADT, a fluoxetina (Baumgartner et al., 1994) também parece induzir a deiodinase II (DII), que aumenta a conversão do T4 em T3 no cérebro. Esta hipótese levou o grupo alemão de pesquisa- dores (Baumgartner, 2000) a sugerir que a ação aumentada destas enzimas (por aumentar a captação tecidual de T4 e a metabolização em T3) seria compatível com as quedas séricas do T4, sem mudanças no T3. O T3, por sua vez, aumentaria a neurotransmissão serotoninérgica e noradrenérgica (Whybrowe e Prange, 1981; Cleare et al., 1995). Assim, é possível que os antidepressivos ISRS aumentem a neurotransmissão serotoninérgica em alguns pacientes deprimidos por, pelo menos, duas diferentes formas. Diretamente por meio do bloqueio da bomba de recaptação e indiretamente pela ativação das enzimas DII, que causa aumento do T3 cerebral, que, por sua vez, inibe (Heal e Smith, 1988; Gur et al., 1999) os auto-receptores pré-sinápticos $5 \mathrm{HT}_{1 \mathrm{~A}^{\prime}}$ promovendo maior liberação de serotonina. Já destacamos que em humanos os níveis séricos de $5 \mathrm{HT}$ se relacionam positivamente com os níveis de T3 (Bahls e Carvalho, 2004).

É necessário cuidado na generalização dos dados com as pesquisas avaliadas anteriormente, pois indicam resultados inconsistentes e algumas vezes controversos. Por exemplo, os três artigos que analisaram os efeitos da fluoxetina (Shelton et al., 1993; Gendall et al., 2003; Gitlin et al., 2004) também apresentaram resultados discrepantes, em relação ao T3; um artigo (Gitlin et al., 2004) apresentou diminuição significativa na décima semana e em outro (Shelton et al., 1993) esta alteração ocorreu na terceira semana, mas normalizou na sexta semana. Quanto ao T4, a fluoxetina em dois artigos (Shelton et al., 1993; Gendall et al., 2003) não causou alterações, e em outro (Gitlin et al., 2004) ocorreu diminuição significativa. Em relação aos respondedores à terapia antidepressiva com fluoxetina, um artigo (Shelton et al., 1993) encontrou diminuição significativa de T3, outro (Gendall et al., 2003) obteve diminuição significativa de T4 e T4L, e outro (Gitlin et al., 2004) não identificou diferenças.

Finalizando, observamos que os estudos com ISRS empregaram diversos desenhos metodológicos. A maioria dos estudos foi de duração entre quatro a seis semanas (Höflich et al., 1992; Shelton et al., 1993; Brady et al., 1994; König et al., 2000; Gendall et al., 2003), havendo dois que avaliaram um tempo maior, de dez (Gitlin et al., 2004) e de 24 semanas (Sagud et al., 2002). Três examinaram pacientes hospitalizados (Höflich et al., 1992; König et al., 2000; Sagud et al., 2002), um em pacientes ambulatoriais (Shelton et al., 1993) e outros três não especificaram o ambiente do estudo (Brady et al., 1994; 2000; Gendall et al., 2003; Gitlin et al., 2004). Um estudo avaliou só mulheres (Sagud et al., 2002), enquanto os outros incluíram ambos os sexos e dois (Shelton et al., 1993; Sagud et al., 2002) incluíram pacientes com alterações prévias nos HT. Quatro pesquisas compararam os ISRS com diferentes ADT - maprotilina (Höflich et al., 1992), desipramina (Shelton et al., 1993), imipramina (Brady et al., 1994) e nortriptilina (Gendall et al., 2003) -, uma usou grupo-controle (Sagud et al., 2002) e uma outra usou grupo-placebo (Brady et al., 1994). Por último, um estudo utilizou dois ISRS (Gitlin et al., 
2004), mas, analisou os resultados em conjunto, não fazendo avaliação isolada de cada antidepressivo nem comparou os resultados entre eles.

A avaliação de possíveis alterações na função tireoidiana na vigência de tratamentos antidepressivos tem recebido vasto interesse na literatura. Joffe et al. (1984) e Baumgartner et al. (1990) postularam que a terapia antidepressiva exerce efeito direto de aumento no metabolismo dos HT no cérebro, e que esta diminuição funcional dos HT está envolvida no mecanismo de ação dos antidepressivos, estando a redução nos níveis de T4 associada à resposta ao tratamento. Como resultado desta revisão, observamos que esta hipótese pode servir para somente uma parcela de pacientes com depressão, pois assim como com os ADT, alguns estudos com ISRS (Shelton et al., 1993; Brady et al., 1994; Sagud et al., 2002) também não confirmaram a diminuição dos níveis plasmáticos de T4 nos respondedores aos antidepressivos.

Uma vez que, na atualidade, os medicamentos antidepressivos da classe dos ISRS são os mais empregados na medicina, se reveste de importância o estudo de seu comportamento sobre os HT. Com o emprego dos medicamentos ADT, a maioria das pesquisas indicou diminuição significativa do T4 e/ou do T4L, dentro dos limites da normalidade e na recuperação do quadro clínico. A literatura que investigou o efeito dos ISRS sobre os HT é pequena e inconsistente. Seus principais achados foram: o TSH não sofreu alteração na maioria das pesquisas e houve tendência à diminuição nos níveis de T4T. Sugerimos, assim como alguns autores (Brady et al., 1994), que os ISRS podem exercer mudanças no eixo tireoidiano de menor impacto que os ADT.

Mesmo nos casos de diminuição plasmática dos HT em resposta à ação dos ISRS, este pode ser um efeito não específico sobre a função tireoidiana (Höflich et al., 1992; König et al., 2000). Existem estudos que encontraram diminuição de T4 e T4L em pacientes com transtorno de pânico respondedores a imipramina e a diazepam (Balon et al., 1991), e diminuição de T4 em esquizofrênicos respondedores aos antipsicóticos (Rinieris et al., 1980; Baumgartner et al., 1988; Roca et al., 1990). Recentemente, Baumgartner (2000) sugere que, caso os HT estejam envolvidos no mecanismo de ação dos antidepressivos, esta ação deve ser secundária a outros efeitos destas substâncias. É mais provável que os HT levem a aumento não específico de vários processos bioquímicos cerebrais, ressaltando que há indícios de que os HT induzem a produção energética mitocondrial (Soboll, 1993; Prengel et al., 2000), favorecendo mudanças na atividade neuronal.

\section{CONCLUSÕES}

Os estudos aqui revisados sugerem que os ISRS promovem efeitos na função tireoidiana de alguns pacientes deprimi- dos, porém esta relação não está suficientemente esclarecida. Clinicamente não conseguimos observar uma aplicação prática desta condição, uma vez que, nem mesmo com os ADT, existe um perfil estabelecido de qual subgrupo de pacientes deprimidos está mais suscetível a mudanças nos seus níveis de HT e se estas alterações trazem repercussões de importância prático-clínica. Talvez o efeito dos ISRS sobre a função tireoidiana possa alcançar relevância no caso de pacientes com patologia tireoidiana. Já citamos um relato de caso (Harel et al., 1995) em adolescente que após 18 meses de uso de sertralina apresentou baixos níveis de T4. E uma série de onze casos (nove pacientes hipotireoideos e dois pacientes com câncer de tireóide) recebendo T4, que após o emprego de sertralina ocorreu aumento de TSH e diminuição de T4, e todos necessitaram de complementação da terapia hormonal (McCowen et al., 1997). Não conhecemos, na literatura nenhum estudo sistematizado sobre prováveis efeitos dos ISRS em pacientes com hipotireoidismo e depressão, representando uma área que necessita de pesquisas que possam indicar sua relevância laboratorial e clínica.

\section{REFERÊNCIAS}

Bahls SC, Carvalho GA. A relação entre a função tireoidiana e a depressão: uma revisão. Rev Bras Psiquiatria. 26:41-9, 2004.

Balon R, Pohl R, Yeragani VK, Ramesh C, Glitz DA. The changes of thyroid hormone during pharmacological treatment of panic disorder patients. Progress in Neuropsychopharmacol Biol Psychiatry. 15:595-600, 1991

Bauer M, Heinz A, Whybrow PC. Thyroid hormones, serotonin and mood: of synergy and significance in the adult brain. Mol Psychiatry. 7:140-56, 2002.

Baumgartner A. Thyroxine and the treatment of affective disorders: an overview of the results of basic and clinical research. Int J Neuropsychopharmacol. 3:149-65, 2000.

Baumgartner A, Dubeyko M, Campos-Barros A, Eravci M, Meinhlod H. Subchronic administration of fluoxetine to rats affects triiodothyronine production and deiodination i regions of the cortex and in the limbic forebrain. Brain Res. 635:68-74, 1994.

Baumgartner A, Gräf K, Kürten I, Meinhold H. The hypothalamic-pituitary-thyroid axis in psychiatric patients and healthy subjects. Psychiatry Res. 24:271-332, 1988.

Baumgartner A, Gräf KJ, Kürten I, Meinhold H, Scholz P. Neuroendocrinological investigations during sleep deprivation. I. Concentrations of thyrotropin, thyroid normones, cortisol, prolactin, luteinizing hormone, follicle stimulating hormone, oestradiol, and testosteron in patients with major depressive disorder at 8 a.m. before and after total sleep deprivation. Biol Psychiatry. 28:556-68, 1990.

Bech P, Kirkegaard C, Boch E, Johannesen M, Rafaelson 0J. Hormones, electrolytes and cerebrospinsl fluid proteins in manic-melancholic patients. Neuropsychobiol. 4:99-112, 1978.

Brady KT, Anton RK. The thyroid axis and desipramine treatment in depression. Biol Psychiatry. 25:703-9, 1989.

Brady KT, Lydiard RB, Kellner CH, Joffe R, Laird LK, Morton WA et al. A comparison of the effects of imipramine and fluvoxamine on the thyroid axis. Biol Psychiatry. 36:778-9, 1994.

Cleare AJ, McGregor A, O'Keane V. Neuroendocrine evidence for an association between hypothyroidism, reduced central 5-HT activity and depression. Clin Endocrinol. 43:713-9, 1995.

Duval F, Mokrani MC, Bailey P, Correa H, Diep TS, Crocq MA, et al. Thyroid axis ativity and serotonin function in major depressive disorder. Psychoneuroendocrinol. 24:695-712, 1999. 
Duval F, Mokrani MC, Crocq MA, Jautz M, Bailey P, Diep TS et al. Effect of antidepressant medication of morning and evening thyroid function tests during a major depressive episode. Arch Gen Psychiatry. 53:833-40, 1996.

Gendall KA, Joyce PR, Mulder RT, Luty SE. Thyroid indices and response to fluoxetine and nortriptyline in major depression. J Psychopharmacol. 17:431-7, 2003.

Gitlin M, Altshuler LL, Frye MA, Suri R, Huynh EL, Fairbanks L et al. Peripheral thyroid hormones and response to selective serotonin reuptake inhibitors. J Psychiatry Neuroscience. 29:383-6, 2004

Gur E, Lerer B, Newman ME. Chronic clomipramine and triiodothyronine increase serotonin levels in rat frontal cortex in vivo: relationship to serotonin autoreceptor activity. J Pharmacol Exp Ther. 288:81-7, 1999.

Harel Z, Biro FM, Tedford WL. Effects of long term treatment with sertraline (Zoloft) simulating hypothyroidism in an adolescent. J Adolesc Health. 16:232-4, 1995.

Heal DJ, Smith SL. The effects of acute and repeated administration of T3 to mice on 5-HT1 and 5 -HT2 function in the brain and its influence on the actions of repeated eletroconvulsive shock. Neuropharmacol. 27:1239-48, 1995.

Höflich G, Kasper S, Danos P, Schmidt R. Thyroid hormones, body temperature, and antidepressant treatment. Biol Psychiatry. 31:859-62, 1992.

Joffe R, Roy-Byrne PP, Udhe TW, Post RM. Thyroid function and affective illness: a reappraisal. Biol Psychiatry. 19:1685-91, 1984.

Joffe RT, Singer W. The effect of tricyclic antidepressants on basal thyroid hormone levels in depressed patients. Pharmacopsychiatry. 23:67-9, 1990.

Joffe RT, Sokolov STH. Thyroid hormones, the brain, and affective disorders. Critical Rev Neurobiol. 8:45-63, 1994.

Kirkegaard C, Bjorum N, Cohn D, Faber J, Birk-Lauridsen U, Nerup J. Studies on the influence of biogenic amines and psychoactive drugs on the prognostic value of the TRH stimulation test in endogenous depression. Psychoneuroendocrinol. 2:131-6, 1977.

König F, Hauger B, von Hippel C, Wolfersdorf M, Kaschka WP. Effect of paroxetine on thyroid levels in severely depressed patients. Neuropsychobiol. 42:135-8, 2000.

Langer G, Schonbeck G, Konig G, Lesch 0, Schussler M, WaldhausI W. Antidepressant drugs and the hypothalamic-pituitary-thyroid axis. Lancet. 1:100-1, 1980.
McCowen KC, Garber JR, Spark R. Elevated serum thyrotropin in thyroxine-treated patients with hypothyroidism given sertraline. N Engl J Med. 337:1010-1, 1997.

Muller B, Boning J. Changes in the pituitary-thyroid axis accompanying major affective disorders. Acta Psychiatr Scan. 77:143-50, 1988.

Prengel H, Brödel 0, Hiedra L, Pinna G, Eravci M, Meinhold H et al. Effects of tranylcipromine on thyroid hormone metabolism and concentrations in rat brain. Neuropharmacol. 39:99$109,2000$.

Rao ML, Ruhrmann S, Retey B, Liappis N, Furger J, Kraemer M, Kasper S, Möller HJ. Low plasma thyroid indices of depressed patients are attenuated by antidepressant drugs and influence treatment outcome. Pharmacopsychiatry. 29:180-6, 1996.

Rinieris P, Christodoulou GN, Souvatzoglou A, Koutras DA, Stefanis C. Free-thyroxine index in schizophrenic patients before and after neuroleptic treatment. Neuropsychobiol. 6:29-33, 1980.

Roca RP, Blackman MR, Ackerley MB, Harman SM, Gregerman RI. Thyroid hormone elevations during acute psychiatric illness: relationship to severely and distinction from hyperthyroidism. Endocrine Res. 16:415-47, 1990.

Sagud M, Pivac N, Mück-Seler D, Jakovljevic M, Mihaljevic-Peles A, Korsic M. Effects of sertraline treatment on plasma cortisol, prolactin and thyroid hormones in famale depressed patients. Neuropsychobiol. 45:139-43, 2002.

Shelton RC, Winn S, Ekhatore N, Loosen PT. The effects of antidepressants on the thyroid axis in depression. Biol Psychiatry. 33:120-6, 1993.

Singhal RL, Rastogi RB, Hrdina PD. Brain biogenic amines and altered thyroid function. Life Sciences. 17:1617-26, 1975.

Soboll S. Thyroid hormone action on mitochondrial energy transfer. Biochimica Biophysica Acta. 1144:1-16, 1993.

Unden F, Ljunggren JG, Kjellman BF, Beck-Friis J, Wetterberg L. Twenty-four-hour serum levels of T4 and T3 in relation to decreased TSH serum levels and decreased TSH response to TRH in affective disorders. Acta Psychiatr Scand. 73:358-65, 1986.

Whybrow PC, Coppen A, Prange AJ, Noguera R, Baily JE. Thyroid function and the response to liothyronine in depression. Arch Gen Psychiatry. 26:242-5, 1972.

Whybrow PC, Prange AJ. A hypothesis of thyroid-catecholamine-receptor interaction. Arch Gen Psychiatry. 38:106-11, 1981. 